\title{
Thermal Decomposition of Cellulose/Synthetic Polymer Blends Containing Grafted Products III. Cellulose/Poly(methyl acrylate) Blends
}

\author{
Noboru Nishioka, ${ }^{\dagger}$ Masayoshi Tabata, Masayuki Saito, Nobuaki Kishigami, \\ Mikio Iwamoto, and Masakuni UNO* \\ Faculty of Engineering Osaka Electro-Communication University, Neyagawa, Osaka 572, Japan \\ * Junior College Division, Osaka Electro-Communication University, \\ Neyagawa, Osaka 572, Japan
}

(Received November 11, 1996)

\begin{abstract}
The homogeneous grafting of methyl acrylate onto cellulose was carried out in a dimethyl sulfoxide/ paraformaldehyde solvent system. The grafted products were added to cellulose/poly(methyl acrylate) (PMA) blends as compatibilizers. The thermal decomposition behavior of the blends was investigated by thermogravimetry. The thermal stability of the blends decreased with an increase in grafted product content. Accessibility determined by a deuteration method increased with grafted product content and peak intensity of wide-angle X-ray diffraction patterns decreased. The microphase-separated structures of the blends with higher grafted product content were finer than those of the blends without grafted products. Dynamic mechanical measurements and differential scanning calorimetry were carried out to determine the glass transition temperatures, $T_{\mathrm{g}}$, of the blends. $T_{\mathrm{g}}$ for cellulose decreased with an increase in grafted product content but that for PMA hardly varied. The difference in thermal decomposition behavior of the blends was correlated to that in compatibility. Thermogravimetry was effective for compatibility estimation in cellulose/PMA blends containing grafted products.
\end{abstract}

KEY WORDS Thermal Decomposition / Cellulosic Blend / Grafted Product / Compatibilizer /

We reported that the thermal decomposition behavior of the vinyl monomer grafted cellulose products depends remarkably on their graft copolymer contents. ${ }^{1,2}$ In grafting, a graft copolymer and attendant homopolymer are synthesized simultaneously. ${ }^{3,4}$ Some of main chains not participating in grafting also remain. The grafted products thus are polymer blends containing graft copolymers as compatibilizers. The difference in thermal stability of the grafted cellulose products was correlated to compatibility. ${ }^{1,2}$ The grafted products with different graft copolymer contents are necessary to correlate thermal stability with compatibility more precisely. In a series of our work, ${ }^{5,6}$ the grafted products themselves were added to the cellulose/synthetic polymer blends as compatibilizers to vary graft copolymer content widely. In this work, methyl acrylate (MA) grafted cellulose products were added to cellulose/poly(methyl acrylate) (PMA) blends.

\section{EXPERIMENTAL}

\section{Grafting and Sample Preparation}

Homogeneous grafting of MA onto cellulose in a dimethyl sulfoxide/paraformaldehyde (DMSO/PF) sol- vent system and characterization of the grafted products were performed by the method described previously. ${ }^{7-10}$ The grafted products are characterized in Table I. In homogeneous grafting, the molecular weight of the graft polymer is presumed to be equal to that of the attendant homopolymer. $^{3,4}$ We confirmed that both molecular weights are consistent with each other within experimental error. ${ }^{8,10}$ The viscosity-average molecular weights, $M_{v}$, of the extracted PMA thus were used as those of the graft polymers. The values of $M_{v}$ of cellulose and PMA used for the blends were estimated to be $146 \times 10^{3}$ and $290 \times 10^{3}$ from the intrinsic viscosities obtained with cadoxen ${ }^{11}$ at $20^{\circ} \mathrm{C}$ and acetone ${ }^{12}$ at $25^{\circ} \mathrm{C}$, respectively.

The crude reaction mixtures and DMSO solutions of cellulose and PMA were mixed to give proper blend ratios. The mixtures were cast onto glass plates at room temperature and dried at about $40^{\circ} \mathrm{C}$ under reduced pressure for $24 \mathrm{~h}$. After being immersed in water for several days, the films were easily peeled off. They were air dried on filter paper at room temperature and stored in a desiccator until use.

Table I. Characterization of grafted products ${ }^{a}$

\begin{tabular}{|c|c|c|c|c|c|c|c|c|}
\hline \multirow{2}{*}{ Sample code } & \multicolumn{3}{|c|}{ PMA content $/ w t \%$} & \multirow{2}{*}{$\begin{array}{c}M_{v} \times 10^{-3} \\
\text { of } \text { graft }^{\mathrm{b}}\end{array}$} & \multirow{2}{*}{$\begin{array}{l}\text { Number } \\
\text { of grafts }\end{array}$} & \multicolumn{3}{|c|}{ Relative number of chains ${ }^{c}$} \\
\hline & Overall & Graft polymer & Homopolymer & & & Cellulose & Graft copolymer & PMA \\
\hline$g$-РМА02 & 46.5 & 11.5 & 35.0 & 71 & 0.44 & 56 & 44 & 134 \\
\hline$g$-РMA05 & 28.7 & 18.7 & 10.0 & 62 & 0.62 & 38 & 62 & 33 \\
\hline$g$-РМА06 & 5.6 & 5.4 & 0.2 & 61 & 0.13 & 87 & 13 & 1 \\
\hline
\end{tabular}

${ }^{\mathrm{a}}$ Viscosity-average molecular weight $\left(M_{v}\right)$ of main chain cellulose is $146 \times 10^{3} .{ }^{\mathrm{b}} M_{v}$ of the extracted PMA homopolymer was used as that of the graft polymer. ${ }^{\mathrm{C}}$ Original number of cellulose chains was assumed to be 100 .

† To whom correspondence should be addressed. 


\section{Measurements}

A SEIKO SSC5000TA with a TG/DTA300 module was used for thermal decomposition measurements. The samples $(c a .15 \mathrm{mg})$ were preheated to $150^{\circ} \mathrm{C}$ at a heating rate of $10^{\circ} \mathrm{C} \mathrm{min}^{-1}$ under nitrogen flow at $100 \mathrm{ml} \mathrm{min}^{-1}$ to evaporate adsorbed water. The measurements were carried out from room temperature to $600^{\circ} \mathrm{C}$ under the conditions described above.

The samples $(c a .5 \times 5 \times 0.02 \mathrm{~mm})$ for accessibility measurement were dried over $\mathrm{P}_{2} \mathrm{O}_{5}$ under reduced pressure for $24 \mathrm{~h}$ at room temperature, immersed in $\mathrm{D}_{2} \mathrm{O}$ for $4 \mathrm{~h}$, and then dried again under reduced pressure for $24 \mathrm{~h}$. Infrared spectra were measured with a JASCO FT/IR-300 spectrometer by placing the samples between $\mathrm{NaCl}$ plates with hexachlorobutadiene. ${ }^{13}$ Accessibility, $\mathrm{A}$, was calculated according to the equation ${ }^{14}$ :

$$
A=100 /\left\{1+1.11\left(A_{\mathrm{OH}} / A_{\mathrm{OD}}\right)\right\}
$$

where $A_{\mathrm{OH}}$ and $A_{\mathrm{OD}}$ are the absorbances at 3360 and $2530 \mathrm{~cm}^{-1}$, respectively.

A Hitachi HU-11A transmission electron microscope (TEM) was used to observe the microphase-separated structures of the samples. The samples were stained with osmic acid vapor for $24 \mathrm{~h}$. The copolymer of $n$-butyl methacrylate and methyl methacrylate was used as the embedding medium. The embedded samples were cut into ultrathin sections of $50 \mathrm{~nm}$ thickness.

Wide angle X-ray diffraction profiles were obtained for the film specimens using a Shimadzu XD-3A diffractometer.

Differential scanning calorimetry (DSC) was carried out with a SEIKO SSC/560S DSC. The samples ( $c a$. $20 \mathrm{mg}$ ) were heated from -50 to $200^{\circ} \mathrm{C}$ at a rate of $20^{\circ} \mathrm{C} \mathrm{min}^{-1}$.

A SEIKO SDM5600H with a DMS200 module was used for dynamic mechanical measurements. The measurements were conducted from -100 to $300^{\circ} \mathrm{C}$ at a frequency of $10 \mathrm{~Hz}$, at a heating rate of $2^{\circ} \mathrm{Cmin}^{-1}$. The samples $(c a .30 \times 6 \times 0.02 \mathrm{~mm})$ were dried at about $40^{\circ} \mathrm{C}$ under reduced pressure for more than $24 \mathrm{~h}$ prior to measurements.

\section{RESULTS AND DISCUSSION}

Figure 1(a) shows the thermogravimetric (TG) curves of the grafted product ( $g$-PMA05) and cellulose/PMA ( $8 / 2$ by weight) blends containing different amounts of $g$-PMA05. The weight of the cellulose $/ g$-PMA05/PMA $(8 / 0 / 2)$ blend without $g$-PMA05 decreases by $55 \%$ at around $300^{\circ} \mathrm{C}$ and then $20 \%$ at around $400^{\circ} \mathrm{C}$, indicating the presence of different thermal decomposition processes. As indicated in the previous work, ${ }^{1,2}$ the processes at lower and higher temperatures result mainly from the decomposition of cellulose and PMA fractions, respectively. The thermal decomposition behavior of the $(8 / 2.1 / 2)$ blend containing 2.1 parts of $g$-PMA05 is similar to that of the $(8 / 0 / 2)$ blend. However, the decomposition of the $(8 / 2.8 / 2)$ blend takes place gradually from around $250^{\circ} \mathrm{C}$. The weight of $g$-PMA05 decreases sharply by $25 \%$ at around $220^{\circ} \mathrm{C}$ and then $45 \%$ at around $400^{\circ} \mathrm{C}$.

The difference in thermal decomposition behavior of these samples can be seen more clearly from the derivative thermogravimetric (DTG) curves in Figure 1(b). The DTG curve of the $(8 / 0 / 2)$ blend has two peaks at 330 and $418^{\circ} \mathrm{C}$. We use these peak temperatures as a measure of thermal stability. The peak temperatures of the $(8 / 2.1 / 2)$ blend are almost equal to those of the $(8 / 0 / 2)$ blend. The peak at lower temperatures of the $(8 / 2.8 / 2)$ blend becomes broad and a small hump appears at around $280^{\circ} \mathrm{C}$. The peak at around $330^{\circ} \mathrm{C}$ of the $(8 / 4.2 / 2)$ blend almost disappears and the peak at around $260^{\circ} \mathrm{C}$ becomes predominant. The DTG curve of $g$-PMA05 shows two peaks at 220 and $416^{\circ} \mathrm{C}$. As mentioned above, the peaks at lower and higher temperatures correspond to the decomposition of cellulose and PMA fractions, respectively. The peak at higher temperatures for these samples hardly shifts, suggesting no influence on the thermal stability of PMA. However, the peak at lower temperatures of $g$-PMA 05 is lower by $110^{\circ} \mathrm{C}$ than that of the $(8 / 0 / 2)$ blend, suggesting the decrease in thermal stability of cellulose.

Figure 1(c) illustrates the differential thermal analysis (DTA) curves of the same samples. Two endothermic peaks associated with the decomposition of cellulose and PMA fractions are observed. ${ }^{1,2}$ We can also use these peak temperatures as a measure of thermal stability. The
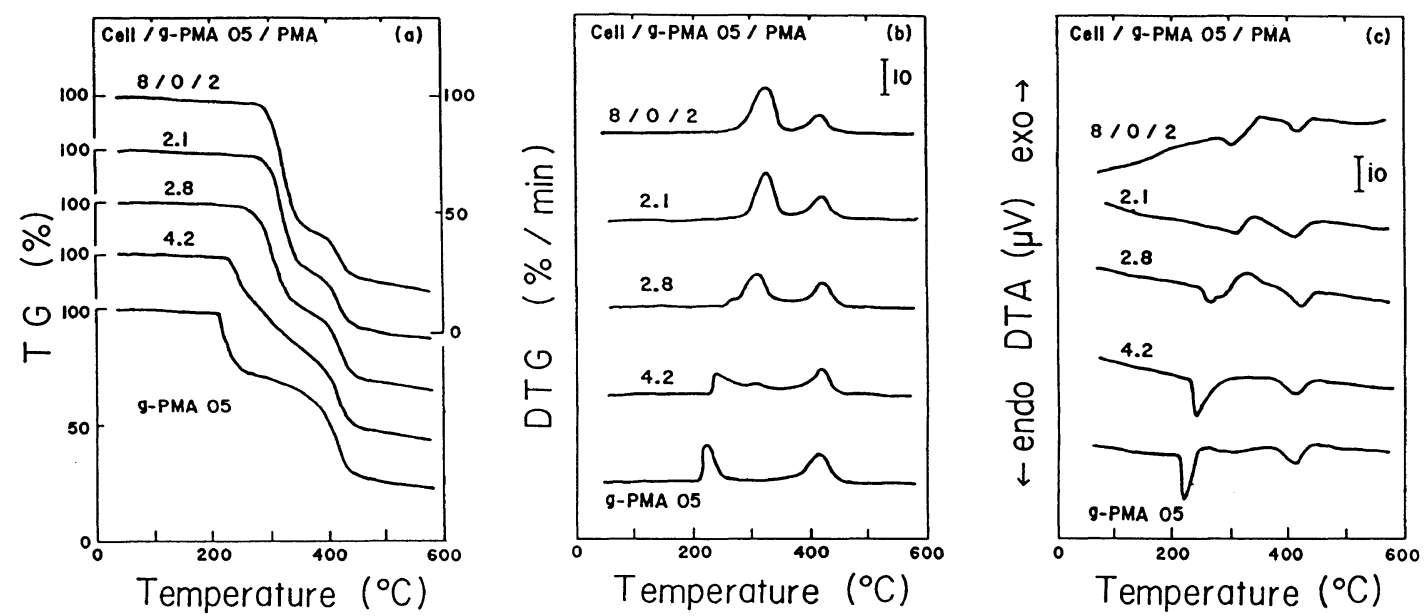

Figure 1. Thermograms of grafted product ( $g$-PMA05) and cellulose/PMA (8/2) blends containing $g$-PMA05: (a) thermogravimetric (TG) curves; (b) derivative thermogravimetric (DTG) curves; (c) differential thermal analysis (DTA) curves. 


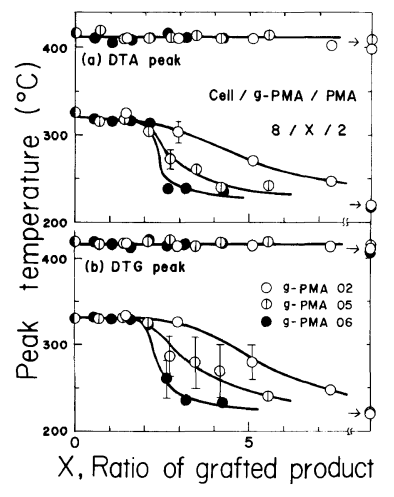

Figure 2. Dependence of DTA and DTG peak temperatures for the cellulose/PMA (8/2) blends containing different grafted products on the grafted product content: $(\bigcirc) g$-PMA02; (D) $g$-PMA05; $g$-PMA06.

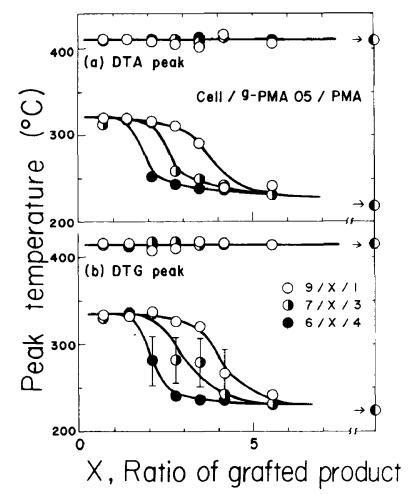

Figure 3. Dependence of DTA and DTG peak temperatures for the cellulose/PMA blends with different compositions on the $g$-PMA05 content: (O) $9 / x / 1$; (○) $7 / x / 3$; (○) $6 / x / 4$; (○) $g$-PMA05.

endothermic peak at lower temperatures shifts toward lower temperatures with the addition of $g$-PMA05 but that at higher temperatures hardly shifts, being consistent with Figure 1(b). It is clear that the increase in $g$-PMA05 content makes the cellulose/PMA blend, especially cellulose, thermally unstable.

The dependence of the DTA and DTG peak temperatures on the grafted product content is revealed in Figure 2. Three grafted products were added to the cellulose/ PMA (8/2) blend. The DTA and DTG peak temperatures indicate a similar dependence on the grafted product content. The peak temperature at around $420^{\circ} \mathrm{C}$ is almost independent of the content and kind of grafted products, while the lower peak temperature decreases at higher contents. The grafted product content required to reduce the thermal stability of the cellulose/PMA (8/2) blend depends on the kind of grafted products. The addition of $g$-PMA06 is most effective of the three grafted products. As characterized in Table I, most of the PMA chains in $g$-PMA06 are grafted onto cellulose chains.

The amounts of $g$-PMA05 required to influence the thermal stability of the cellulose/PMA blends with different compositions are compared in Figure 3. The peak temperature at around $420^{\circ} \mathrm{C}$ hardly depends on the $g$-PMA05 content and cellulose/PMA composition, being similar to the result in Figure 2. On the other hand, the lower peak temperature decreases with an increase in $g$-PMA05 content. The cellulose/PMA (6/4) blend is

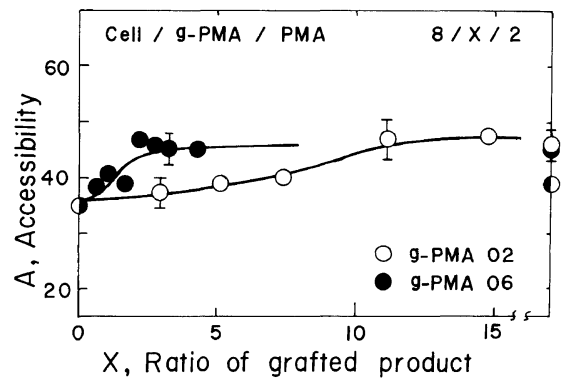

Figure 4. Dependence of accessibility for cellulose/PMA (8/2) blends containing different grafted products on the grafted product content: (O) $g$-PMA02; (๑) $g$-PMA06; () cellulose/PMA (8/2); (O) cellulose.

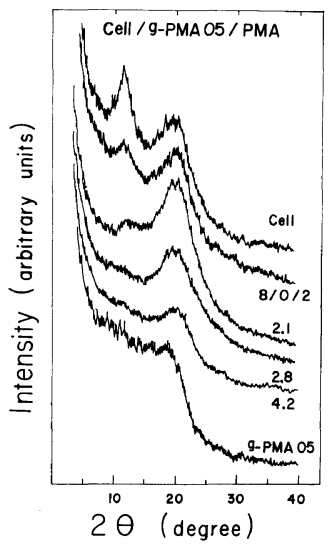

Figure 5. X-Ray diffraction patterns of cellulose, cellulose/PMA (8/2) blends containing $g$-PMA05, and $g$-PMA05.

most sensitive to the addition of $g$-PMA05. It is of interest to note that the grafted product content affecting the thermal stability of the cellulose/PMA blends depends on both the kind of grafted products and cellulose/PMA composition. Moreover, it should be noted that the thermal stability of cellulose in the blends is mainly influenced.

In Figure 4, the value of accessibility determined by the deuteration method is plotted against the grafted product content. Accessibility is defined as the ratio of the number of hydroxyl groups reacted with $\mathrm{D}_{2} \mathrm{O}$ to that of total hydroxyl groups. Accessibility is a measure of the crystallinity of cellulose, higher accessibility corresponding to lower crystallinity. ${ }^{14-16}$ The values of the grafted products are larger than that ${ }^{17}$ of cellulose cast from a DMSO/PF solution, implying that the PMA chains hinder the crystallization of cellulose chains to result in an increase in free hydroxyl groups. The accessibility increases with grafted product content and approaches that of grafted products. Such an increase in accessibility corresponds to a decrease in peak temperature for cellulose shown in Figure 2.

Figure 5 indicates the X-ray diffraction patterns of cellulose, cellulose/PMA (8/2) blends containing $g$ PMA05, and $g$-PMA05. The peak intensity of the blends decreases with an increase in $g$-PMA05 content, suggesting a decrease in crystallinity. This is consistent with an increase in accessibility. Thermal stability of polymers mainly depends on crystallinity. ${ }^{18-20}$ Any change in thermal stability is not expected for amorphous PMA. Therefore, the decrease in thermal stability of the blends is attributable to that in crystallinity of cellulose. 

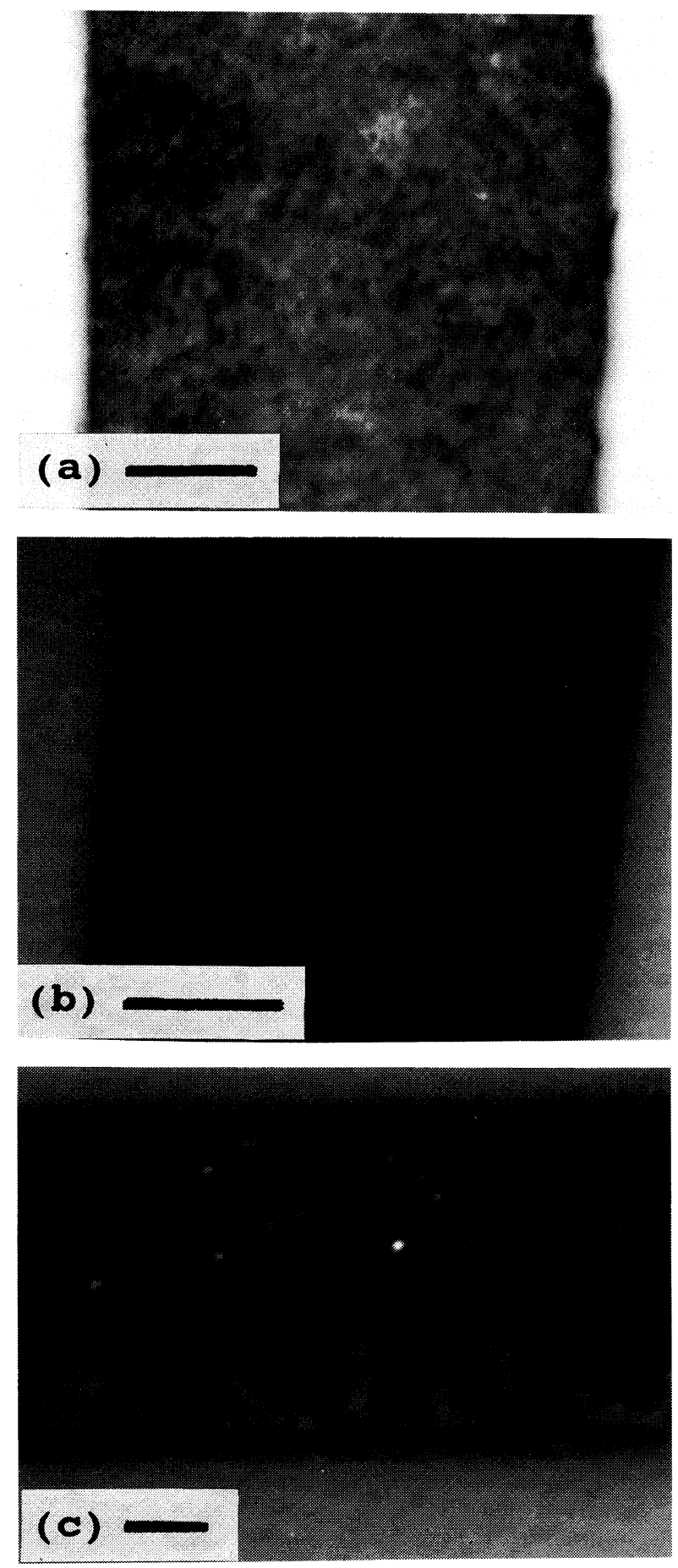

Figure 6 shows TEM micrographs of $g$-PMA02 and cellulose/PMA $(8 / 2)$ blends containing $g$-PMA02. The dark domains indicate the cellulose phases stained with osmic acid. The microphase-separated structure of $g$ PMA02 is very fine, indicating good compatibility of cellulose with PMA. The dark domains in the $(8 / 0 / 2)$ blend are relatively large, those in the $(8 / 2.9 / 2)$ blend small, and those in the $(8 / 5.2 / 2)$ blend faint. The change in microphase-separated structures with the addition of $g$-PMA02 is obvious. A comparison of TEM micrographs with thermal decomposition behavior indicates that the thermal stability of the blends with fine microphaseseparated structures decreases remarkably. The fine microphase-separated structure, large accessibility, and
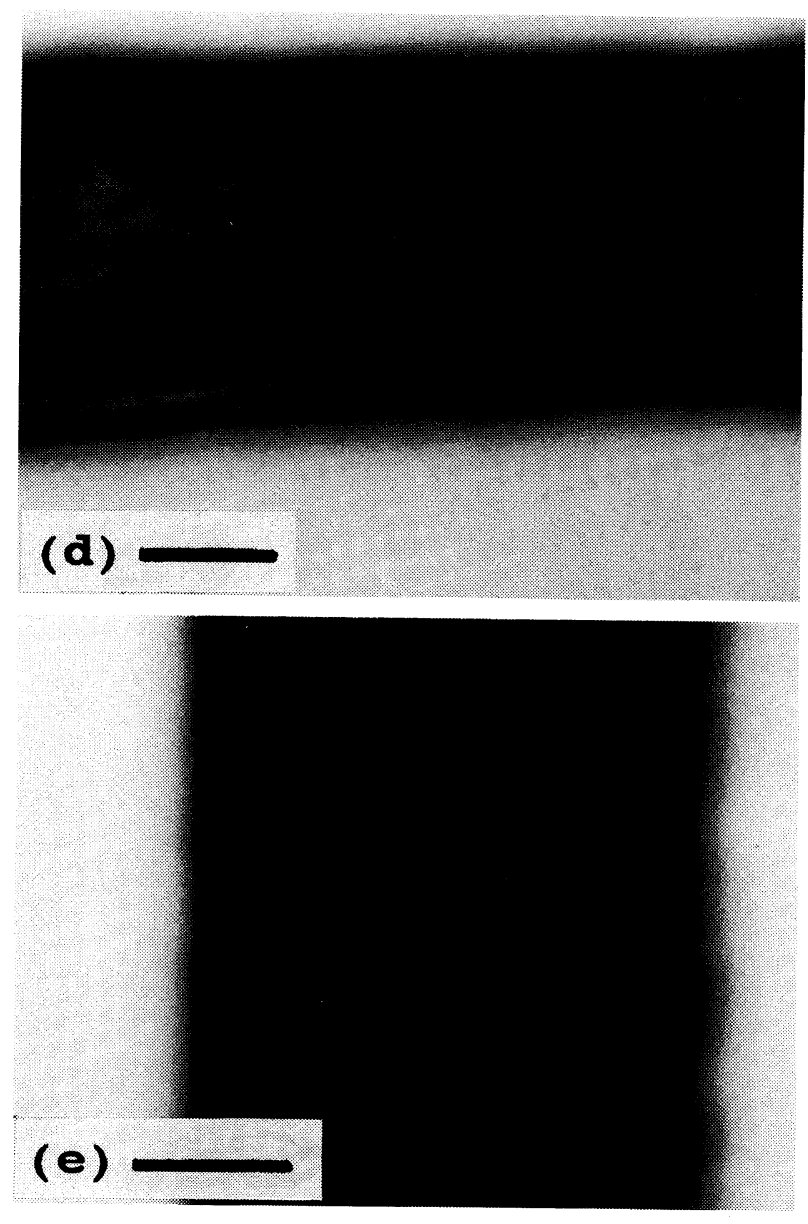

Figure 6. TEM micrographs of $g$-PMA02 and cellulose/PMA (8/2) blends containing $g$-PMA02. Scale bar represents $5 \mu \mathrm{m}$ : (a) $g$-PMA02; (b) $8 / 0 / 2$; (c) $8 / 2.9 / 2$; (d) $8 / 5.2 / 2$; (e) $8 / 7.4 / 2$.

low diffraction intensity imply good compatibility of cellulose with PMA in the blend. The thermal stability of the blends with good compatibility may thus decreases remarkably. In this work, the word "compatibility" does not refer to miscibility on a molecular level.

The miscibility in polymer blends is estimated from glass transition temperatures, $T_{\mathrm{g}} \cdot{ }^{21-23}$ However, cellulose does not undergo a clear glass transition. DSC measurements were performed. The glass transition at lower temperatures around $20^{\circ} \mathrm{C}$ for PMA was obvious, but that at higher temperatures for cellulose was not clear. A similar difficulty in determining $T_{\mathrm{g}}$ by DSC for blends containing cellulose has been reported. ${ }^{1,5,6,24-27}$

The temperature dependence of the mechanical loss 


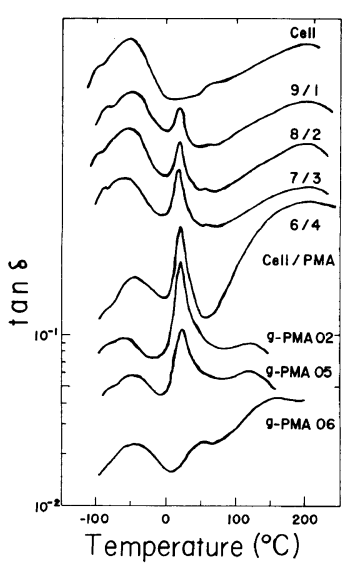

Figure 7. Temperature dependence of mechanical loss tangent for cellulose, cellulose/PMA blends with different compositions, and grafted products.

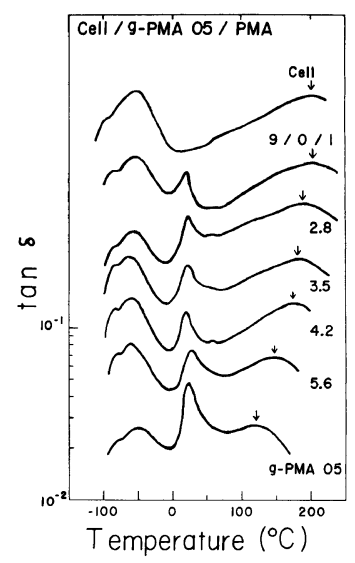

Figure 8. Temperature dependence of mechanical loss tangent for cellulose/PMA (9/1) blends containing $g$-PMA05.

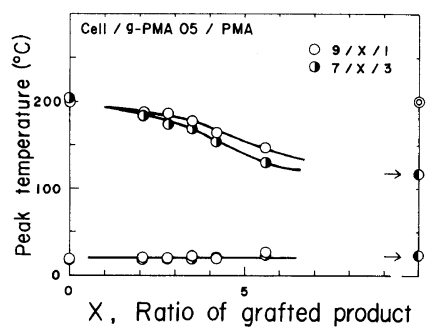

Figure 9. Dependence of the peak temperatures of mechanical loss tangent for cellulose/PMA blends with different compositions on the

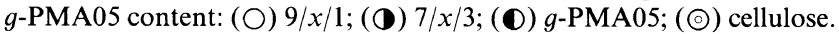

tangent for cellulose, cellulose/PMA blends, and grafted products is given in Figure 7. The cellulose sample indicates two broad relaxation peaks. The peak at around $-50^{\circ} \mathrm{C}$ is assigned to relaxation caused by the motion of methylol groups in the glucose residues. ${ }^{28-31}$ The peak at around $200^{\circ} \mathrm{C}$ corresponds to the glass transition of cellulose main chains. A similar glass transition peak has been reported by Masson and Manley. ${ }^{27}$ They used a cellulose film cast from a DMSO/PF solution and reported that the peak temperature is possibly the $T_{\mathrm{g}}$ of cellulose lowered by the presence of residual methylol substituents. The blends indicate three relaxation peaks. The sharp peak at around $20^{\circ} \mathrm{C}$ is assigned to the glass transition of PMA. In spite of the change in composition, both glass transition peaks hardly shift, indicating that cellulose is immiscible with PMA. The grafted products also indicate three relaxation peaks. The glass transition peak for cellulose shifts to lower temperatures, indicating the influence on the molecular motion of cellulose chains. The relaxation peaks for PMA of $g$-PMA02 and $g$ PMA05 hardly shift, but that of $g$-PMA06 shifts to higher temperatures. As can be seen from Table I, most of the PMA chains are grafted onto cellulose chains.

Figure 8 also indicates the temperature dependence of the mechanical loss tangent for the cellulose/PMA (9/1) blends containing $g$-PMA05. $T_{\mathrm{g}}$ for cellulose decreases gradually with an increase in $g$-PMA05 content, but that for PMA hardly shifts. The $g$-PMA05 content dependence of these peak temperatures is shown in Figure 9. In both blends, $T_{\mathrm{g}}$ for cellulose decrease with an increase in $g$-PMA05 content. It is clear that the molecular motion of cellulose chains is influenced by the addition of $g$-PMA05. The decrease in $T_{\mathrm{g}}$ for cellulose corresponds to that in the DTG and DTA peak temperatures shown in Figure 3. Thus, the variation in thermal stability of the blends is correlated to that in compatibility of them.

As indicated previously, ${ }^{1}$ the depression of thermal stability was not found for the miscible amorphous polymer blend of poly(vinyl chloride) with PMA. The compatibility estimation by thermogravimetry may be a characteristic method for blends containing cellulose as one component. Several miscible cellulose/synthetic polymer blends have been reported..$^{24-27,32}$ The thermal decomposition of these miscible blends was investigated. ${ }^{33}$ One blend indicated a similar decrease in thermal stability but other blends did not. The difference in thermal decomposition of the cellulosic blends must be closely correlated to that in compatibility. Moreover, the relationship between the compatibilizing effect of graft copolymers and structure must be elucidated.

\section{REFERENCES}

1. N. Nishioka, H. Ono, and M. Karasuno, Polym. J., 24, 301 (1992).

2. N. Nishioka and N. Yoshida, Polym. J., 24, 1009 (1992).

3. S. M. Hudson and J. A. Cuculo, J. Macromol. Sci., Rev. Macromol. Chem., C18, 1 (1980).

4. A. Hebeish and J. T. Guthrie, "The Chemistry and Technology of Cellulosic Copolymers," Springer-Verlag, Berlin-HeidelbergNew York, 1981.

5. N. Nishioka, M. Yamaoka, H. Haneda, K. Kawakami, and M. Uno, Macromolecules, 26, 4694 (1993).

6. N. Nishioka, Y. Nakano, T. Hirota, N. Fujiwara, and M. Uno, J. Appl. Polym. Sci., 59, 1203 (1996).

7. N. Nishioka and K. Kosai, Polym. J., 13, 1125 (1981).

8. N. Nishioka, K. Matsumoto, and K. Kosai, Polym. J., 15, 153 (1983).

9. N. Nishioka, K. Minami, and K. Kosai, Polym. J., 15, 591 (1983).

10. N. Nishioka, Y. Matsumoto, T. Yumen, K. Monmae, and K. Kosai, Polym. J., 18, 323 (1986).

11. G. Jayme and P. Kleppe, Papier, 15, 492 (1961).

12. M. Guaita and O. Chiantore, Makromol. Chem., 176, 185 (1975).

13. H. Tadokoro, "Structure of Crystalline Polymers," Wiley, New York, N.Y., 1979.

14. J. Mann and H. J. Marrinan, Trans. Faraday Soc., 52, 492 (1956).

15. J. Mann, "Cellulose and Cellulose Derivatives," Vol. V, Part IV, N. M. Bikales and L. Segal, Ed., Wiley, New York, N.Y., 1971, p 89.

16. M. Ioelovitch and M. Gordeev, Acta Polym., 45, 121 (1994).

17. N. Nishioka, K. Watase, K. Arimura, K. Kosai, and M. Uno, Polym. J., 16, 867 (1984). 
18. A. Basch and M. Lewin, J. Polym. Sci., Polym. Chem. Ed., 11, 3071 (1973).

19. M. E. Calahorra, M. Cortázar, J. I. Eguiazábal, and G. M. Guzmán, J. Appl. Polym. Sci., 37, 3305 (1989).

20. L. Reich and S. S. Stivala, "Elements of Polymer Degradation," McGraw-Hill, New York, N.Y., 1971.

21. D. R. Paul and S. Newman, Ed., "Polymer Blends," Academic Press, New York, N.Y., 1978

22. O. Olabisi, L. M. Robeson, and M. T. Schaw, "Polymer-Polymer Miscibility," Academic Press, New York, N.Y., 1979.

23. L. A. Utracki, "Polymer Alloys and Blends," Carl Hanser Verlag, Munich/FRG, 1989

24. Y. Nishio, S. K. Roy, and R. St. J. Manley, Polymer., 28, 1385 (1987).

25. Y. Nishio and R. St. J. Manley, Macromolecules, 21, 1270 (1988).
26. Y. Nishio, N. Hirose, and T. Takahashi, Polym. J., 21, 347 (1989).

27. J-F. Masson and R. St. J. Manley, Macromolecules, 24, 6670 (1991).

28. Y. Ishida, M. Yoshino, M. Takayanagi, and F. Irie, J. Appl. Polym. Sci., 1, 227 (1959).

29. M. Kimura and J. Nakano, J. Polym. Sci., Polym. Lett. Ed., 14, 741 (1976).

30. D. J. Crofton and R. A. Pethrick, Polymer, 22, 1048 (1981).

31. D. J. Crofton, D. Mancrieff, and R. A. Pethrick, Polymer, 23, 1605 (1982).

32. J-F. Masson and R. St. J. Manley, Macromolecules, 24, 5914 (1991).

33. N. Nishioka, S. Hamabe, H. Murakami, and T. Kitagawa, to appear. 